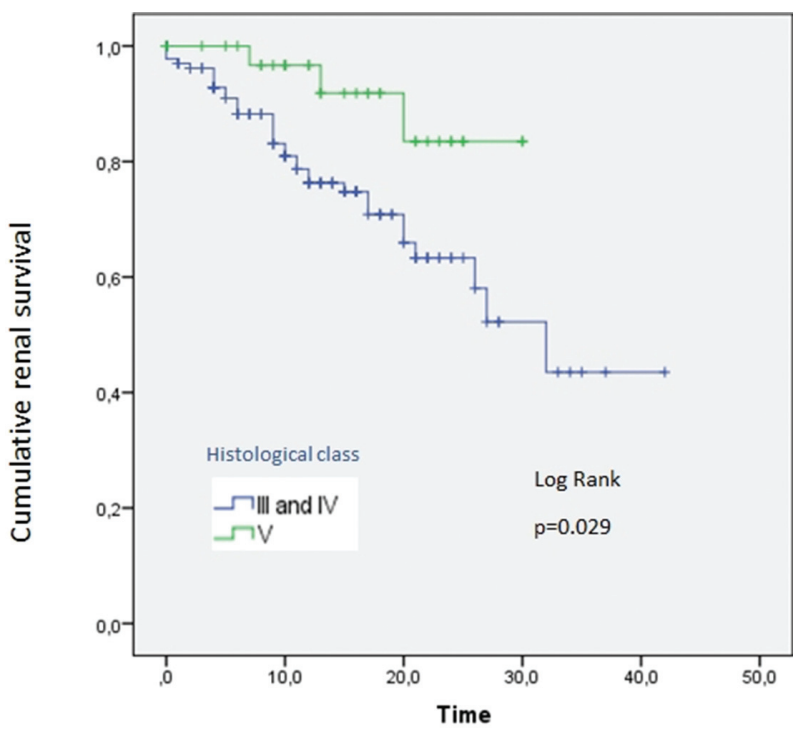

Conclusions: In spite of presenting in the context of the same autoimmune systemic disease, PLN and MLN appear to be very different entities, showing significant differences regarding serologic profiles and renal survival.

Disclosure of Interest: None declared

DOI: 10.1136/annrheumdis-2018-eular.5433

\section{SAT0433 APPLICATION OF THE DORIS ALGORITHM FOR THE DEFINITION OF DISEASE REMISSION OVER A 2-YEAR PERIOD IN A COHORT OF ITALIAN PATIENTS WITH SYSTEMIC LUPUS ERYTHEMATOSUS}

F. Dall'ara ${ }^{1}$, L. Andreaoli ${ }^{1}$, G. Armentaro ${ }^{1}$, F. Migliorati $^{1}$, M. Frassi $^{1}$, F. Franceschini ${ }^{1}$, S. Calza ${ }^{2}$, A. Tincani ${ }^{1} .{ }^{1}$ Rheumatology and Clinical Immunology, Department of Clinical and Experimental Sciences, Spedali Civili and University of Brescia; ${ }^{2}$ Department of Translational and Molecular Medicine, Biostatistics Unit, University of Brescia, Brescia, Italy

Background: Systemic Lupus Erythematosus (SLE) is characterised by a fluctuating course. To achieve sustained remission is the ultimate goal of maintenance treatment. However remission is difficult to define in SLE. In 2014, an international Task Force named DORIS proposed four definitions of remission. ${ }^{1}$

Objectives: Aim of this study was to evaluate the performance of the DORIS algorithm in comparison to the remission status as defined by clinical judgement and to identify the frequency of remission as determined by DORIS for each clinical disease pattern.

Methods: Monocentric retrospective study. Among all SLE patients followed at the Lupus Clinic between 2014 and 2016, we enrolled patients fulfilling the SLICC 2012 criteria who were visited at least once in 2016 and who had at least 5 biannual medical examinations in the previous 2 years. Definitions of remission according to DORIS, Clinical Remission, and disease patterns are reported in table 1.

Results: 101 SLE patients were enrolled for this study (94\% female, mean age 45 years). $17.8 \%$ of patients were in remission in all the 5 time-points, vice versa $29.7 \%$ of patients never got into remission. $17.8 \%$ of patients have been in remission for 24 months, while $21.8 \%$ of patients less then 6 months. Mean duration of DORIS remission was 7.96 months. The most prevalent disease patter were RR $(41,6 \%)$ and CQ $(41,6 \%)$, while CA pattern was present in $16,8 \%$ of patients. DORIS remission was most frequently achieved in CQ pattern (65.2\% of visits), less frequently in CA (5.9\%). 294 visits out of $505(58 \%)$ were defined as "nonremission" according to DORIS. cSLEDAl above zero was the item that most frequently accounted for "non-remission", particularly urinary and haematological (as reported in figure 1). In 229 (43.3\%) visits there was a disagreement between DORIS and clinical judgement: the reasons for discordant results were respectively: a) self-management of steroids dosage and precautionary increase of steroids in the suspect of a flare in $7.1 \%$; b) cSLEDAI $>0$ in $27.2 \%, P G A \geq 0.5$ in $12.6 \%$, more than one of these items in $53.1 \%$.
Abstract SAT0433 - Table 1. Definition of Remission according to Doris, Definition of Clinical Remission according to Clinical judgement; Definition of disease pattern. PGA: Physician Global Assessment; cSLEDAI: clinical SLEDAI

\begin{tabular}{|c|c|c|c|c|c|}
\hline \multirow[t]{2}{*}{ Definition of Remi ssion } & \multicolumn{2}{|c|}{ Clinical Items } & \multicolumn{3}{|c|}{ Treatment } \\
\hline & CSLEDAI & PGA & $\begin{array}{l}\text { Daily dose of } \\
\text { prednisone }\end{array}$ & Antimalarials & $\begin{array}{c}\text { Immunosuppressants/ } \\
\text { Biologics }\end{array}$ \\
\hline Remission "off treatment" & $=0$ & $<0.5$ & 0 & yes & no \\
\hline Remission "on treatment" & $=0$ & $<0.5$ & $\leq 5 \mathrm{mg}$ & yes & yes \\
\hline No Remission & $\neq 0$ & 20.5 & $25 \mathrm{mg}$ & yes & yes \\
\hline $\begin{array}{l}\text { Definition of Clinical judgement } \\
\text { of Remission }\end{array}$ & \multicolumn{5}{|c|}{$\begin{array}{l}\text { Ab sence of any incr ease in corticosteroids dosage or any change in } \\
\text { immunosuppressants }\end{array}$} \\
\hline \multicolumn{6}{|l|}{ Definition of disease patterns } \\
\hline Chronic active (CA) & \multicolumn{5}{|c|}{$\begin{array}{l}\text { Persistent dis ease activity over tim e with a CSLEDAI } 1 \text { in each visit for at least } \\
\text { one year }\end{array}$} \\
\hline Relapsing-remitting (RR) & \multicolumn{5}{|c|}{$\begin{array}{l}\text { Characterised by periods of disease activity with CSLEDAl } \geq 1 \text { interspersed with } \\
\text { periods of disease inactivity with cSLEDAI }=0 \text { in different visits for at least one } \\
\text { year }\end{array}$} \\
\hline Clinical quiescent (CQ) & \multicolumn{5}{|c|}{ Defined as absence of disease activity with a cSLEDAl = 0 for at least one year } \\
\hline
\end{tabular}

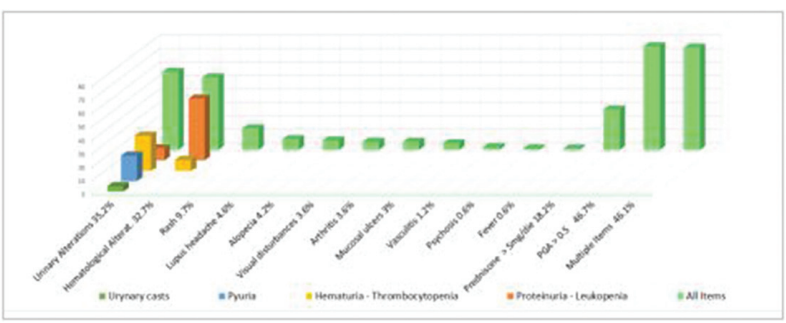

Abstract SAT0433 - Figure 1. Items are responsible of disagreement between DORIS and Clinical definition of Remission

Conclusions: Nearly $40 \%$ of the visits displayed a disagreement between clinical judgement of remission and DORIS remission. This may be attributable mainly to a different approach in evaluating patients: longitudinal by clinical judgement and cross-sectional by DORIS. As compared to clinical judgement of remission, the DORIS definition is not designed to capture "low disease activity", particularly patients who carry a PGA between 0.5 and 1 and those who require a medium dosage of steroids in the frame of a CA pattern.

\section{REFERENCE:}

[1] van Vollenhoven R, et al. Ann Rheum Dis 2017 Mar;76(3):554-561.

Disclosure of Interest: None declared

DOI: 10.1136/annrheumdis-2018-eular.6480

\section{SAT0434 \\ PARTICIPATION IN SYSTEMIC LUPUS ERYTHEMATOSUS - A CROSS-SECTIONAL ANALYSIS OF THE LULA-COHORT IN GERMANY 2015}

G. Chehab $^{1}$, J.G. Richter ${ }^{1}$, R. Fischer-Betz ${ }^{1}$, R. Brinks ${ }^{1}$, B. Winkler-Rohlfing ${ }^{2}$, M. Schneider'. ${ }^{1}$ Policlinic of Rheumatology and Hiller Research Unit Rheumatology, Medical Faculty, Heinrich-Heine-University, Duesseldorf, ${ }^{2}$ German Lupus Self-Help Community, Wuppertal, Germany

Background: Participation of chronically ill patients can be permanently and severely impaired. In a heterogeneous disease like systemic lupus erythematosus (SLE), various influencing factors such as disease activity, damage, concomitant diseases, but also detrimental effects due to the psychological burden must be considered.

Objectives: Our objective was to assess the current state of participation in a representative sample of German patients with SLE and evaluate the impact of demographic and clinical factors.

Methods: The Lupus erythematosus long-term study (LuLa-Study), a nationwide longitudinal study among German Caucasian patients with SLE, is being 
conducted annually by a self-reported questionnaire since 2001. Inclusion criteria are a diagnosis of SLE and returning the completed paper questionnaire. Amongst others medication, comorbidities, health-related quality of life (HRQoL, Short-Form-12), and disease activity (Systemic Lupus Activity Measure) are surveyed. In the year 2015 we additionally inquired about participation using the "Index zur Messung von Einschränkungen der Teilhabe" (IMET; Index for measuring limitations of participation) that was developed on the basis of the International Clas sification of Functioning, Disability and Health (ICF), as well as depression (Centre for Epidemiologic Studies Depression Scale), and pain coping (Pain Related Self statements scale).

A multiple linear regression was run to predict overall impairment and impairment in the individual subdomains (dependent variables). Age, disease duration, number of comorbidities, pain, disease activity, catastrophizing, coping, depression, physical functioning, and physical and mental HRQoL were entered into our model as candidates for the independent variables. Variable selection was accomplished by a stepwise approach based on Akaike information criterion (AIC).

Results: The questionnaire was completed by 579 patients (response rate $89.2 \%)$. Only $48(8.3 \%)$ reported no impairment of participation by their disease. Most limitations were reported in the domains 'stress and extraordinary strains' (56.3\% reported moderate to high impairment) and 'sex life' (48.7\%), whereas 'common activities of daily life' (21.1\%) and 'close personal relationships' $(26.5 \%)$ seemed to be limited less frequently.

Depression, physical functioning, and physical and mental HRQoL predicted overall participation (F-test, $p<0.0001$ ).

Depression, physical functioning, and physical HRQoL predicted also most of the participation subdomains whereas age, disease duration, no. of comorbidities, disease activity, pain, and pain coping behaviour impacted only individual subdomains.

Conclusions: Limitations of participation are common in SLE patients and affect different areas of life. In order to improve participation, it is of great importance to maintain, respectively improve physical and mental quality of life, physical functioning, and depression. The direction of causality cannot be proved beyond reasonable doubt in this cross-sectional analysis. Additional longitudinal studies are necessary.

Acknowledgements: The LuLa-study is supported by unrestricted grants from GlaxoSmithKline and UCB Pharma

Disclosure of Interest: $G$. Chehab Grant/research support from: GlaxoSmithKline and UCB Pharma for performing the LuLa-study., J. Richter Grant/research support from: GlaxoSmithKline and UCB Pharma for performing the LuLa-study., R. Fischer-Betz Grant/research support from: GlaxoSmithKline and UCB Pharma for performing the LuLa-study., R. Brinks: None declared, B. Winkler-Rohlfing: None declared, M. Schneider Grant/research support from: GlaxoSmithKline and UCB Pharma for performing the LuLa-study.

DOI: 10.1136/annrheumdis-2018-eular.3229

\section{SAT0435 \\ PLASMA PTX3 LEVELS CORRELATE WITH SYSTEMIC LUPUS ERYTHEMATOSUS ACTIVITY AND ARE INFLUENCED BY CORTICOSTEROIDS}

G.A. Ramirez ${ }^{1,2}$, E.P. Bozzolo ${ }^{2}$, V. Canti $^{2}$, B. Bottazzi ${ }^{3}$, A. Mantovani ${ }^{3,4}$ L. Dagna ${ }^{1,2}$, P. Rovere-Querini ${ }^{1,2}$, A.A. Manfredi ${ }^{1,2}$. ${ }^{1}$ Università Vita-Salute San Raffaele; ${ }^{2}$ Unit of Immunology, Rheumatology Allergy and Rare Diseases, IRCCS San Raffaele Hospital, Milan; ${ }^{3}$ IRCCS Humanitas Research Hospital; ${ }^{4}$ Humanitas University, Rozzano (MI), Italy

Background: Systemic lupus erythematosus (SLE) is an autoimmune disease characterised by a variable involvement of multiple organs and tissues. Acute and/or chronic vascular inflammation is not uncommon in patients with SLE and can significantly affect patient quality of life and survival. Specific markers of vascular inflammation in SLE are lacking. Pentraxin-3 (PTX3) is an evolutionarily conserved pattern recognition receptor expressed by multiple cell lines and is growingly recognised as a marker of the vessel response to injury. Glucocorticoids are known inducers of PTX3 in most tissues. The role of PTX3 as a biomarker in SLE is discussed.

Objectives: To assess the potential informative role of PTX3 as a biomarker in patients with SLE with and without current or previous vasculitic manifestations and with active or quiescent disease.

Methods: We enrolled 55 adult patients with SLE for a total of 60 samples. Samples were classified as taken from patients with active disease (SLE disease activity index, SLEDAI, $\geq 4$ ) with or without active vasculitis and from patients with quiescent disease (SLEDAI <4). Further stratification was performed according to a history of lupus vasculitis. Five patients were bled twice under different conditions. Plasma PTX3 was measured by ELISA. Non-parametric tests were employed to compare PTX3 levels among groups.

Results: PTX3 plasma levels were slightly but not significantly more elevated in patients with active vasculitis. PTX3 levels correlated with SLEDAI in the whole set of patients $(p=0.007)$ and in those who were off corticosteroids $(p<0.001)$, but not in patients receiving prednisone. PTX3 levels correlated with the dose of prednisone $(p<0.001)$. Patients with $>1$ moderately-to-highly active $(A, B)$ British Isles Lupus Assessment Group (BILAG) domain had significantly higher PTX3 levels than those with more limited disease activity extent $(p=0.041)$. PTX3 also correlated with a 0.0-3.0 physician global assessment scale (PGA), with patientreported visual analogue scale, and inversely with $C 4$ levels $(p=0.004, p=0.013$, $\mathrm{p}=0.001$ respectively). There was no significant correlation with age or disease duration nor with C-reactive protein (CRP). Similar to PTX3, CRP was higher in patients with $>1$ A/B BILAG domain $(p=0.004)$, but did not correlate with SLEDA or prednisone dose. Repeated samples showed a high intra-individual variability for PTX3, which unpredictably correlated with disease activity and prednisone dosage.

Conclusions: Our data suggest that PTX3 is a marker of active disease extent rather than vascular inflammation in SLE and it shares this behaviour with CRP, another member of the pentraxin family. Nonetheless, PTX3 also specifically cor relate with monoparametric indexes of activity such as SLEDAI. A high intra-individual variability and the effect of corticosteroids constitute potential limitations to future diagnostic applications of PTX3 in SLE.

\section{REFERENCES:}

[1] Erreni M, et al. Immunol Rev 2017

[2] Skare T, et al. Joint Bone Spine 2015.

[3] Assandri R, et al. Autoimmune Diseases 2015

Disclosure of Interest: None declared

DOI: 10.1136/annrheumdis-2018-eular.7317

\section{SAT0436 TRANSJUGULAR RENAL BIOPSY: A SAFE AND EFFECTIVE WAY TO PERFORM RENAL BIOPSY IN SYSTEMIC LUPUS ERYTHEMATOSUS AND ANTIPHOSPHOLIPID ANTIBODY SYNDROME PATIENTS TREATED WITH ANTI-THROMBOTIC DRUGS - A MONOCENTRIC EXPERIENCE OF 256 PROCEDURES}

H. Nielly ${ }^{1}$, A. Mathian ${ }^{1,2,3}$, M. Cazenave ${ }^{1}$, H. Izzedine ${ }^{4}$, J. Haroche ${ }^{1,2,3}$, F. CohenAubart $^{1,2,3}$, P. Rouvier ${ }^{5}$, I. Brocheriou ${ }^{3,5}$, P. Cluzel ${ }^{3,6}$, Z. Amoura $^{1,2,3}{ }^{1}{ }^{1}$ French national reference center for systemic lupus erythematosus and antiphospholipid antibody syndrome, service de Médecine interne 2, Institut E3M, Assistance Publique-Hôpitaux de Paris (AP-HP), Groupement Hospitalier Pitié-Salpêtrière (GHPS); ${ }^{2}$ U1135, Centre d'Immunologie et des Maladies Infectieuses (CIMI-Paris), INSERM; ${ }^{3}$ Université Pierre et Marie Curie, Université Pierre et Marie Curie; ${ }^{4}$ service de néphrologie, Assistance Publique-Hôpitaux de Paris (AP-HP), Groupement Hospitalier Pitié-Salpêtrière (GHPS); ${ }^{5}$ Service d'Anatomie et Cytologie Pathologiques, Assistance Publique-Hôpitaux de Paris (AP-HP), Groupement Hospitalier Pitié-Salpétrière; ${ }^{6}$ Département d'imagerie cardiovasculaire et de radiologie interventionnelle, Assistance Publique-Hôpitaux de Paris (AP-HP), Groupement Hospitalier Pitié-Salpêtrière (GHPS), Paris, France

Background: Renal biopsy is the cornerstone of Lupus nephritis (LN) management. However, transcutaneous renal biopsy (TCRB) is hampered by the antithrombotic treatment frequently prescribed in Systemic Lupus Erythematosus (SLE) and Antiphospholipid Antibody Syndrome (APS). Transjugular renal biopsy (TJRB) offers an attractive alternative for patients at increased risk of bleeding. Objectives: The primary objective of the study was to describe the safety and the diagnostic performance of TJRB in SLE and APS.

Methods: A retrospective review of SLE and/or APS patients who consecutively underwent a renal biopsy in our department between January 2004 and October 2016 was performed. Biopsies were divided into four groups: TCRB, TJRB with aspirin treatment (aspirin TJRB), TJRB with anticoagulant treatment (anticoagulant TJRB), and TJRB without anti-thrombotic drug (no-antithrombotic TJRB). Major complications were defined as decease, haemostasis nephrectomy, renal artery embolization, blood transfusion, sepsis and vascular thrombosis. Minor complications were defined as gross haematuria, renal hematoma and arteriovenous fistula.

Results: Fifty-four TCRB and 256 TJRB were analysed - 69 aspirin TJRB, 68 anticoagulant TJRB and 119 no-antithrombotic TJRB. Major complications rate was $1.9 \%$ for TCRB and $2.0 \%$ for TJRB $(p=1)$. One patient in the TJRB group suffering from catastrophic antiphospholipid syndrome (CAPS) died suddenly 6 weeks after the biopsy. No patient died of bleeding complication. One patient in the anticoagulant TJRB group required a renal artery embolization and blood transfusion. Four other patients required blood transfusion (1 in the TCRB group 1 in the aspirin TJRB group and 2 in the anticoagulant TJRB group). Minor complications rate was $1.9 \%$ for TCRB and $7.8 \%$ for TJRB $(p=0.2)$.

Among the 256 TJRB, the rate of complication (major or minor) was higher for patients with glomerular filtration rate CKD-EPI $<30 \mathrm{~mL} / \mathrm{min}(6 / 24$ [25\%]) compared to patients with GFR $>30 \mathrm{~mL} / \mathrm{min}(16 / 232$ [7\%], $\mathrm{p}<0.01$ using the Khi-2 test). Age over 40, blood pressure $>140 / 90 \mathrm{mmHg}$, APS or positive 